A Novel Highly Selective Chiral Auxiliary for the Asymmetric Synthesis of L- and D- $\alpha$-Amino Acid Derivatives via Multicomponent Ugi Reaction

Andrea Basso, Luca Banfi, Renata Riva and Giuseppe Guanti

Università degli Studi di Genova

Dipartimento di Chimica e Chimica Industriale

Via Dodecaneso 31, 16146 Genova (Italy)

\title{
Content List
}

General remarks

General procedures

$\mathrm{S} 2$

Detailed compound characterization

S3

\section{GENERAL REMARKS}

\section{NMR spectra}

${ }^{1}$ H-N.M.R.s have been recorded on a VARIAN "MERCURY 300” at $300 \mathrm{MHz}$. Chemical shifts are reported in p.p.m. using TMS $(0.00$ ppm) as internal standard. J constants are reported in Hz.

${ }^{13}$ C N.M.R.s have been recorded on a VARIAN “MERCURY 300" at $75 \mathrm{MHz}$.

When not indicated, spectra have been recorded at room temperature in $\mathrm{CDCl}_{3}$ as the solvent.

\section{GC-MS analysis}

GC-MS analyses have been performed on a HP-5890 seriies II with a HP-1 column (530 $\mu$, lenght $12 \mathrm{~m}$, internal diameter $0.2 \mathrm{~mm}$ ). Ultrapure helium has been used as carrier gas. Mass spectra (electron impact) were recorded on a HP-5971A spectrometer, coupled to the above chromatograph. Conditions for the GC-MS analyses are as follow:

flow: $0.9 \mathrm{~mL} / \mathrm{min}$; initial temperature: $100^{\circ} \mathrm{C}$; initial time: $2 \mathrm{~min}$; rate: $20^{\circ} \mathrm{C} / \mathrm{min}$; final temperature: $260^{\circ} \mathrm{C}$; final time: 4 min.; injector temperature: $250^{\circ} \mathrm{C}$

\section{TLC analysis (Thin Layer Cromatography)}

All the analyses have been performed on silica TLC plates MERCK 60 F254 (0.25 mm thick). Spots have been observed under the UV light $(\lambda=254 \mathrm{~nm})$ or stained with an aqueous solution of $\mathrm{KMnO}_{4}$ or with a solution of $\left(\mathrm{NH}_{4}\right) \mathrm{MoO}_{4} \bullet 4 \mathrm{H}_{2} \mathrm{O}$ and $\mathrm{Ce}\left(\mathrm{SO}_{4}\right)_{2}$ in diluited $\mathrm{H}_{2} \mathrm{SO}_{4}$ or with iodine vapours.

\section{Cromatographic purifications}

Flash cromatographies have been performed on ICN Biomedicals 60A silica (230-400 mesh)

\section{Optical rotations}

Optical rotations were determined with a Jasco DP-181 polarimeter, using a Jasco cyclindrical cell $10 \times 100 \mathrm{~mm}$.

\section{Elemental analyses}

Microanalyses were performed on a Heraeus CHN-O-Rapid instrument. 


\section{Solvents}

Anhydrous methylene chloride, N,N-dimethylformamide, methanol, ethanol and acetonitrile have been bought by FLUKA or ALDRICH.

Anhydrous tetrahydrofuran has been freshly distilled over potassium under a nitrogen atmosphere. Petroleum ether, ethyl acetate, methylene chloride, diethyl ether and triethylamine have been distilled or bought with a high purity grade.

\section{Reagents}

All reagents have been bought from FLUKA or ALDRICH

\section{GENERAL PROCEDURES}

\section{General procedure for the Ugi reaction}

The amino acid $(0.29 \mathrm{mmol})$ was suspended in methanol $(1 \mathrm{~mL})$ and aldehyde $(0.32 \mathrm{mmol})$ and isocyanide $(0.32 \mathrm{mmol})$ were added in sequence. The reaction was stirred at room temperature under nitrogen for 2-5 days, then concentrated in vacuo and purified by flash chromatography

\section{General procedure for the retro Diels-Alder reaction}

The bicyclic derivative $(0.20 \mathrm{mmol})$ was dissolved in toluene $(5 \mathrm{~mL})$ and heated at reflux for 2 hours. The solvent was then removed under reduced pressure yielding the desired enamine derivative

\section{General procedure for the enamine cleavage}

The enamine $(0.20 \mathrm{mmol})$ was dissolved in methylene chloride and treated with $\mathrm{HCl}$ (4M solution in dioxane, $0.15 \mathrm{~mL}$ ) for 2 hours. The solvents were then evaporated and the crude material dissolved in methylene chloride and neutralised with a saturated solution of sodium bicarbonate. The organic phase was concentrated and purified by flash chromatography.

\section{General procedure for the $N$-benzyl cleavage}

The substrate $(0.20 \mathrm{mmol})$ was dissolved in a $4.4 \%$ solution of formic acid in methanol $(1 \mathrm{~mL})$, then palladium black $(50 \mathrm{mg})$ was added and the resulting sospension stirred under nitrogen at room temperature overnight. The catalyst was then filtered and washed with methanol $(1 \mathrm{~mL})$, the solvent was then removed under reduced pressure and the crude product purified by flash chromatography.

\section{General procedure for the preparation of the Mosher's amides}

Amino acid benzylamide $(0.03 \mathrm{mmol})$ was dissolved in methylen chloride $(0.2 \mathrm{~mL})$ together with triethylamine $(20 \mu \mathrm{L})$. (R)- or $(\mathrm{S})$-Mosher's chloride $(0.06 \mathrm{mmol}, 15 \mu \mathrm{L})$ was then added and the reaction left agitating for 24 hours. The solution was then diluited with ethyl acetate $(5 \mathrm{~mL})$ and extracted with HCL $1 \mathrm{~N}(3 \times 1 \mathrm{~mL})$. The organic phase was concentrated in vacuo and a ${ }^{1} \mathrm{H}-\mathrm{NMR}$ spectrum was recorded on the crude material. The crude material was then purified via preparative TLC plate.

\section{DETAILED COMPOUND CHARACTERIZATION (compd. 9a-g, 11a-g, 12a-g)}

\section{Compound (9a)}

M.W.: 448.55

R.f. $=0.65$ (eluent: $\mathrm{EtOAc} / \mathrm{PE}=1: 1$ )

$\left.[\alpha]_{\mathrm{D}}{ }^{20}:+53.7(c) 1.38, \mathrm{CHCl}_{3}\right)$ 
${ }^{1} \mathbf{H}$ NMR (300 MHz): $\delta 0.89$ [3H, d, $J$ 7]; 1.12 [3H, d, $J$ 7]; 2.10-2.25 [1H, m]; 2.85 [1H, dd, $\left.J ~ 5,3\right]$; $2.88[1 \mathrm{H}, \mathrm{d}, J 10] ; 3.34[1 \mathrm{H}, \mathrm{d}, J 3] ; 3.56[1 \mathrm{H}, \mathrm{d}, J 16] ; 3.57[3 \mathrm{H}, \mathrm{s}] ; 4.32[1 \mathrm{H}, \mathrm{dd}, J 14,5] ; 4.50$ $[1 \mathrm{H}, \mathrm{d}, J 16] ; 4.59[1 \mathrm{H}, \mathrm{dd}, J 14,8] ; 4.64\left[1 \mathrm{H}, \mathrm{dt}, J_{\mathrm{d}} 4, J_{\mathrm{t}} 1\right] ; 4.86[1 \mathrm{H}, \mathrm{dd}, J 1.5,0.6] ; 6.24[1 \mathrm{H}, \mathrm{dd}$, $J 6,1] ; 6.32$ [1H, dd, $J 6,2] ; 6.54[1 \mathrm{H}, \mathrm{t}, J 6] ; 7.20-7.40[10 \mathrm{H}, \mathrm{m}]$.

${ }^{13}$ C NMR $(75 \mathrm{MHz}): \delta 19.8\left(\mathrm{CH}_{3}\right) ; 20.5\left(\mathrm{CH}_{3}\right) ; 27.8(\mathrm{CH}) ; 43.2\left(\mathrm{CH}_{2}\right) ; 46.1(\mathrm{CH}) ; 51.7\left(\mathrm{CH}_{3}\right)$ and $\left(\mathrm{CH}_{2}\right) ; 63.8(\mathrm{CH}) ; 68.1(\mathrm{CH}) ; 78.4(\mathrm{CH}) ; 83.8(\mathrm{CH}) ; 126.5(\mathrm{CH}) ; 127.5(\mathrm{CH}) ; 128.0(\mathrm{CH}) ; 128.1$ $(\mathrm{CH}) ; 128.3(\mathrm{CH}) ; 128.8(\mathrm{CH}) ; 135.4(\mathrm{CH}) ; 136.2(\mathrm{CH}) ; 138.3(\mathrm{C}) ; 140.2(\mathrm{C}) ; 172.1(\mathrm{C}) ; 172.4(\mathrm{C})$. elem. anal. calc. for $\mathrm{C}_{27} \mathrm{H}_{32} \mathrm{~N}_{2} \mathrm{O}_{4}$ : C, 72.30, H, 7.19, N, 6.25; found $\mathrm{C}, 72.38, \mathrm{H}, 7.26, \mathrm{~N}, 6.24$.

\section{Compound (11a)}

M.W.: 296.41

R.f. $=0.49$ (eluent: $\mathrm{DCM} / \mathrm{MeOH}=95: 5$ )

$[\alpha]_{\mathrm{D}}{ }^{20}:-22.7\left(c 1.20, \mathrm{CHCl}_{3}\right)$

${ }^{1}$ H NMR $(300 \mathrm{MHz}): \delta 0.98[6 \mathrm{H}, \mathrm{d}, J$ 7]; 2.20-2.40 [1H, m]; 3.36 [1H, broad s]; 3.85 [2H, dd, $J$ 24, 13]; 4.40-4.55 [2H, m]; 7.20-7.40 [10H, m]; 8.00 [1H, broad s].

${ }^{13}$ C NMR (75 MHz): $817.9\left(\mathrm{CH}_{3}\right) ; 19.5\left(\mathrm{CH}_{3}\right) ; 31.2(\mathrm{CH}) ; 43.1\left(\mathrm{CH}_{2}\right) ; 53.3\left(\mathrm{CH}_{2}\right) ; 67.8(\mathrm{CH})$; $127.3(\mathrm{CH}) ; 127.4(\mathrm{CH}) ; 127.7(\mathrm{CH}) ; 128.3(\mathrm{CH}) ; 128.5(\mathrm{CH}) ; 128.6(\mathrm{CH}) ; 138.5(\mathrm{C}) ; 138.7(\mathrm{C})$; $172.8(\mathrm{C})$.

GC-MS $9.53 \min (100 \%)$ [296 (2\%, M), 162 (90\%), 91 (100\%)].

\section{Compound (12a)}

M.W.: 206.28

R.f. $=0.09$ (eluent: $\mathrm{DCM} / \mathrm{MeOH}=95: 5$ )

$[\alpha]_{\mathrm{D}}{ }^{20}:-23.3\left(c 0.70, \mathrm{CHCl}_{3}\right)$

${ }^{1} \mathbf{H}$ NMR (300 MHz): $\delta 0.84[3 \mathrm{H}, \mathrm{d}, J$ 7]; 0.99 [3H, d, $J$ 7]; 1.67 [2H, broad s]; 2.30-2.40 [1H, m]; $3.30[1 \mathrm{H}, \mathrm{d}, J$ 4]; $4.43[1 \mathrm{H}, \mathrm{dd}, J 15,5] ; 4.48[1 \mathrm{H}, \mathrm{dd}, J 15,6] ; 7.20-7.40[5 \mathrm{H}, \mathrm{m}] ; 7.70$ [1H, broad s].

${ }^{13}$ C NMR (75 MHz): $\delta 16.1\left(\mathrm{CH}_{3}\right) ; 19.7\left(\mathrm{CH}_{3}\right) ; 30.8(\mathrm{CH}) ; 43.0\left(\mathrm{CH}_{2}\right) ; 60.1(\mathrm{CH}) ; 127.3(\mathrm{CH})$; $127.7(\mathrm{CH}) ; 128.6(\mathrm{CH}) ; 138.6(\mathrm{C}) ; 174.1(\mathrm{C})$.

GC-MS $7.03 \min (100 \%)$ [206 (<1\%, M), 91 (100\%), $72(60 \%)]$.

\section{Compound (9b)}

M.W.: 462.58

R.f. $=0.56$ (eluent: $\mathrm{EtOAc} / \mathrm{PE}=3: 7$ )

$[\alpha]_{\mathrm{D}}{ }^{\mathbf{2 0}}:+48.3\left(c 1.08, \mathrm{CHCl}_{3}\right)$

${ }^{1}$ H NMR (300 MHz): $\delta 0.90[3 \mathrm{H}, \mathrm{d}, J$ 6]; 0.97 [3H, d, $J$ 6]; 1.46-1.60 [1H, m]; 1.75-2.00 [2H, m]; $2.91[1 \mathrm{H}, \mathrm{t}, J$ 4]; $3.26[1 \mathrm{H}, \mathrm{d}, J$ 4]; $3.41[1 \mathrm{H}, \mathrm{dd}, J 8,6] ; 3.55[3 \mathrm{H}, \mathrm{s}] ; 3.99[2 \mathrm{H}, \mathrm{s}] ; 4.33[1 \mathrm{H}, \mathrm{dd}, J$ $15,5] ; 4.48[1 \mathrm{H}, \mathrm{dd}, J 15,6] ; 4.86[1 \mathrm{H}, \mathrm{d}, J 4] ; 4.90[1 \mathrm{H}$, broad s]; $6.32[1 \mathrm{H}, \mathrm{dd}, J 6,1] ; 6.40[1 \mathrm{H}$, $\mathrm{dd}, J 6,2] ; 7.12[1 \mathrm{H}, \mathrm{t}, J 6] ; 7.20-7.40[10 \mathrm{H}, \mathrm{m}]$.

${ }^{13}$ C NMR $(75 \mathrm{MHz}): \delta 22.5\left(\mathrm{CH}_{3}\right) ; 23.1\left(\mathrm{CH}_{3}\right) ; 25.7(\mathrm{CH}) ; 38.2\left(\mathrm{CH}_{2}\right) ; 43.2\left(\mathrm{CH}_{2}\right) ; 47.4(\mathrm{CH}) ; 51.0$ $\left(\mathrm{CH}_{2}\right) 51.9\left(\mathrm{CH}_{3}\right) ; 59.7(\mathrm{CH}) ; 64.3(\mathrm{CH}) ; 78.2(\mathrm{CH}) ; 82.6(\mathrm{CH}) ; 127.0(\mathrm{CH}) ; 127.3(\mathrm{CH}) ; 127.7$ $(\mathrm{CH}) ; 128.1(\mathrm{CH}) ; 128.3(\mathrm{CH}) ; 128.6(\mathrm{CH}) ; 135.7(\mathrm{CH}) ; 135.9(\mathrm{CH}) ; 138.5(\mathrm{C}) ; 139.8(\mathrm{C}) ; 172.3$ (C); $173.2(\mathrm{C})$.

elem. anal. calc. for $\mathrm{C}_{28} \mathrm{H}_{34} \mathrm{~N}_{2} \mathrm{O}_{4}$ : $\mathrm{C}, 72.70, \mathrm{H}, 7.41, \mathrm{~N}, 6.06$; found $\mathrm{C}, 72.66, \mathrm{H}, 7.33, \mathrm{~N}, 5.89$.

\section{Compound (11b)}

M.W.: 310.43

R.f. $=0.10$ (eluent: PE/EtOAc $=6: 4$ )

$[\alpha]_{\mathrm{D}}{ }^{20}:-15.9\left(c 0.60, \mathrm{CHCl}_{3}\right)$

${ }^{1}$ H NMR (300 MHz): $\delta 0.84$ [3H, d, $J$ 6]; 0.93 [3H, d, $J$ 6]; 1.40-1.80 [4H, m]; 3.23 [1H, dd, $J$ 9, 4]; 
$3.69[2 \mathrm{H}, \mathrm{dd}, J 31,13] ; 4.45[2 \mathrm{H}, \mathrm{d}, J 6] ; 7.10-7.40[10 \mathrm{H}, \mathrm{m}] ; 7.58[1 \mathrm{H}$, broad s].

${ }^{13}$ C NMR (75 MHz): $\delta 21.7\left(\mathrm{CH}_{3}\right) ; 23.3\left(\mathrm{CH}_{3}\right) ; 25.2(\mathrm{CH}) ; 43.0\left(\mathrm{CH}_{2}\right) ; 43.1\left(\mathrm{CH}_{2}\right) ; 53.0\left(\mathrm{CH}_{2}\right) ; 61.0$ $(\mathrm{CH}) ; 127.3(\mathrm{CH}) ; 127.4(\mathrm{CH}) ; 127.6(\mathrm{CH}) ; 128.1(\mathrm{CH}) ; 128.6(\mathrm{CH}) ; 128.7(\mathrm{CH}) ; 138.6(\mathrm{C}) ; 139.5$ (C); $174.7(\mathrm{C})$.

GC-MS $9.83 \min (100 \%)$ [310 (1\%, M), $176(100 \%), 91(70 \%)]$.

Compound (12b)

M.W.: 220.31

$[\alpha]_{\mathbf{D}}{ }^{20}:+6.8(c 1.45, \mathrm{MeOH})$

${ }^{1} \mathbf{H}$ NMR $\left(300 \mathrm{MHz}, \mathrm{CDCl}_{3}\right): \delta 0.94[3 \mathrm{H}, \mathrm{d}, J$ 6]; $0.97[3 \mathrm{H}, \mathrm{d}, J$ 6]; 1.20-1.80 [5H, m]; 3.40-3.50 $[1 \mathrm{H}, \mathrm{m}] ; 4.43[2 \mathrm{H}, \mathrm{d}, J 6] ; 7.20-7.40[5 \mathrm{H}, \mathrm{m}] ; 7.66[1 \mathrm{H}$, broad s].

${ }^{13}$ C NMR $\left(75 \mathrm{MHz}, \mathrm{CDCl}_{3}\right): \delta 21.3\left(\mathrm{CH}_{3}\right) ; 23.4\left(\mathrm{CH}_{3}\right) ; 24.9(\mathrm{CH}) ; 43.1\left(\mathrm{CH}_{2}\right) ; 44.1\left(\mathrm{CH}_{2}\right) ; 53.5$ $(\mathrm{CH}) ; 127.3(\mathrm{CH}) ; 127.7(\mathrm{CH}) ; 128.6(\mathrm{CH}) ; 138.6(\mathrm{C}) ; 175.4(\mathrm{C})$.

GC-MS $7.23 \min (100 \%)$ [220 (<1\%, M), $91(20 \%), 86(100 \%)]$.

\section{Compound (9c)}

M.W.: 434.53

R.f. $=0.34$ (eluent: $\mathrm{EtOAc} / \mathrm{PE}=3: 7$ )

$[\alpha]_{\mathrm{D}}{ }^{20}:+44.8\left(c 0.90, \mathrm{CHCl}_{3}\right)$

${ }^{1}$ H NMR (300 MHz): $\delta 1.08$ [3H, t, $J$ 7]; 1.70-1.85 [1H, m]; 1.85-2.00 [1H, m]; $2.91[1 \mathrm{H}, \mathrm{t}, J$ 4]; $3.28[1 \mathrm{H}, \mathrm{d}, J 4] ; 3.29[1 \mathrm{H}, \mathrm{dd}, J 7,6] ; 3.55[3 \mathrm{H}, \mathrm{s}] ; 3.94[1 \mathrm{H}, \mathrm{d}, J 15] ; 4.06[1 \mathrm{H}, \mathrm{d}, J 15] ; 4.36[1 \mathrm{H}$, dd, $J 15,6] ; 4.47[1 \mathrm{H}, \mathrm{dd}, J 15,6] ; 4.87\left[1 \mathrm{H}, \mathrm{dt}, J_{\mathrm{d}} 4, J_{\mathrm{t}} 1\right] ; 4.90[1 \mathrm{H}$, broad s]; $6.32[1 \mathrm{H}, \mathrm{dd}, J 6,1]$; 6.38 [1H, dd, $J 6,2] ; 7.08[1 \mathrm{H}, \mathrm{t}, J 6] ; 7.20-7.40[10 \mathrm{H}, \mathrm{m}]$.

${ }^{13}$ C NMR $(75 \mathrm{MHz}): \delta 12.5\left(\mathrm{CH}_{3}\right) ; 22.3\left(\mathrm{CH}_{2}\right) ; 43.2\left(\mathrm{CH}_{2}\right) ; 47.3(\mathrm{CH}) ; 51.2\left(\mathrm{CH}_{2}\right) ; 51.8\left(\mathrm{CH}_{3}\right) ; 63.5$ $(\mathrm{CH}) ; 64.3(\mathrm{CH}) ; 78.2(\mathrm{CH}) ; 82.6(\mathrm{CH}) ; 126.9(\mathrm{CH}) ; 127.3(\mathrm{CH}) ; 127.8(\mathrm{CH}) ; 128.2(\mathrm{CH}) ; 128.3$ $(\mathrm{CH}) ; 128.6(\mathrm{CH}) ; 135.8(\mathrm{CH}) ; 136.0(\mathrm{CH}) ; 138.5(\mathrm{C}) ; 139.8(\mathrm{C}) ; 172.3(\mathrm{C}) ; 173.0(\mathrm{C})$.

elem. anal. calc. for $\mathrm{C}_{26} \mathrm{H}_{30} \mathrm{~N}_{2} \mathrm{O}_{4}$ : C, 71.87, H, 6.96, N, 6.45; found $\mathrm{C}, 72.08, \mathrm{H}, 7.12, \mathrm{~N}, 6.44$.

\section{Compound (11c)}

M.W.: 282.38

R.f. $=0.10$ (eluent: $\mathrm{PE} / \mathrm{EtOAc}=7: 3$ )

$[\alpha]_{\mathrm{D}}{ }^{20}:-21.4\left(c 1.55, \mathrm{CHCl}_{3}\right)$

${ }^{1}$ H NMR $(300 \mathrm{MHz}): \delta 0.94[3 \mathrm{H}, \mathrm{t}, J$ 7]; 1.55-1.90 [3H, m]; $3.15[1 \mathrm{H}, \mathrm{dd}, J$ 7, 5]; $3.71[2 \mathrm{H}, \mathrm{dd}, J$ 21, 13]; 4.45 [2H, d, $J$ 6]; 7.20-7.40 [10H, m]; 7.57 [1H, broad s].

${ }^{13}$ C NMR (75 MHz): $\delta 10.2\left(\mathrm{CH}_{3}\right) ; 26.7\left(\mathrm{CH}_{2}\right) ; 43.0\left(\mathrm{CH}_{2}\right) ; 52.9\left(\mathrm{CH}_{2}\right) ; 63.8(\mathrm{CH}) ; 127.3(\mathrm{CH})$; $127.4(\mathrm{CH}) ; 127.6(\mathrm{CH}) ; 128.1(\mathrm{CH}) ; 128.5(\mathrm{CH}) ; 128.7(\mathrm{CH}) ; 138.6(\mathrm{C}) ; 139.5(\mathrm{C}) ; 174.0(\mathrm{C})$.

GC-MS $9.39 \min (100 \%)$ [148 (90\%), $91(100 \%)]$.

\section{Compound (12c)}

M.W.: 192.26

$[\alpha]_{\mathrm{D}}{ }^{20}:+12.2(c 1.20, \mathrm{MeOH})$

${ }^{1} \mathbf{H}$ NMR $\left(300 \mathrm{MHz}, \mathrm{CDCl}_{3}\right): \delta 0.97$ [3H, t, $J$ 7]; 1.43 [2H, broad s]; 1.50-2.00 [2H, m]; 3.35 [1H, d, $J$ 8, 4]; 4.44 [2H, ddd, $J 21,15,6] ; 7.20-7.40[5 \mathrm{H}, \mathrm{m}] ; 7.60$ [1H, broad s].

${ }^{13} \mathrm{C} \mathrm{NMR}\left(75 \mathrm{MHz}, \mathrm{CDCl}_{3}\right): \delta 10.0\left(\mathrm{CH}_{3}\right) ; 28.1\left(\mathrm{CH}_{2}\right) ; 43.0\left(\mathrm{CH}_{2}\right) ; 56.4(\mathrm{CH}) ; 127.3(\mathrm{CH}) ; 127.7$ $(\mathrm{CH}) ; 128.6(\mathrm{CH}) ; 138.6(\mathrm{C}) ; 174.8(\mathrm{C})$.

GC-MS $6.51 \min (100 \%)$ [192 (<1\%), $91(20 \%), 58(100 \%)]$.

\section{Compound (9d)}

M.W.: 512.60

R.f. $=0.20$ (eluent: $\mathrm{EtOAc} / \mathrm{PE}=3: 7$ ) 
$[\alpha]_{\mathrm{D}}{ }^{20}:+48.5\left(c 0.94, \mathrm{CHCl}_{3}\right)$

${ }^{1}$ H NMR (300 MHz): $\delta 3.00[1 \mathrm{H}, \mathrm{t}, J$ 4]; $3.46[1 \mathrm{H}, \mathrm{d}, J$ 4]; $3.51[1 \mathrm{H}, \mathrm{m}] ; 3.59[3 \mathrm{H}, \mathrm{s}] ; 3.63[1 \mathrm{H}, \mathrm{d}$, $J$ 15]; $3.81[3 \mathrm{H}, \mathrm{s},] ; 4.26[2 \mathrm{H}$, broad d, $J 15] ; 4.53[1 \mathrm{H}, \mathrm{s}] ; 4.86[1 \mathrm{H}, \mathrm{dd}, J 15,8] ; 4.95\left[1 \mathrm{H}, \mathrm{dt}, J_{d} 4\right.$, $\left.J_{t} 1\right] ; 6.09[1 \mathrm{H}, \mathrm{dd}, J 6,1] ; 6.29[1 \mathrm{H}, \mathrm{dd}, J 6,1] ; 6.78[1 \mathrm{H}, \mathrm{t}, J 2] ; 6.88[1 \mathrm{H}, \mathrm{d}, J 8] ; 6.91[1 \mathrm{H}, \mathrm{m}, \mathrm{Ar}]$; 7.20-7.40 [11H, m]; $8.24[1 \mathrm{H}, \mathrm{dd} J 8,4]$.

${ }^{13}$ C NMR (75 MHz): $\delta 43.1\left(\mathrm{CH}_{2}\right) ; 47.2(\mathrm{CH}) ; 50.2\left(\mathrm{CH}_{2}\right) ; 52.1\left(\mathrm{CH}_{3}\right) ; 55.2\left(\mathrm{CH}_{3}\right) ; 65.1(\mathrm{CH}) ; 67.1$ $(\mathrm{CH}) ; 77.7(\mathrm{CH}) ; 80.2(\mathrm{CH}) ; 113.1(\mathrm{CH}) ; 116.5(\mathrm{CH}) ; 122.5(\mathrm{CH}) ; 127.1(\mathrm{CH}) ; 127.4(\mathrm{CH}) ; 128.4$ $(\mathrm{CH}) ; 128.5(\mathrm{CH}) ; 128.7(\mathrm{CH}) ; 129.6(\mathrm{CH}) ; 135.3(\mathrm{CH}) ; 136.7(\mathrm{CH}) ; 137.4(\mathrm{C}) ; 138.5(\mathrm{C}) ; 138.8$; 159.7 (C); 170.7 (C); $172.7(\mathrm{C})$.

elem. anal. calc. for $\mathrm{C}_{31} \mathrm{H}_{32} \mathrm{~N}_{2} \mathrm{O}_{5}$ : C, 72.64, $\mathrm{H}, 6.29, \mathrm{~N}, 5.47$; found $\mathrm{C}, 72.46, \mathrm{H}, 6.26, \mathrm{~N}, 5.28$.

\section{Compound (11d)}

M.W.: 360.45

R.f. $=0.12$ (eluent: $\mathrm{PE} / \mathrm{EtOAc}=1: 1$ )

$[\alpha]_{\mathbf{D}}{ }^{20}:+59.8\left(c 1.14, \mathrm{CHCl}_{3}\right)$

${ }^{1}$ H NMR (300 MHz): $\delta 2.03$ [1H, broad s]; $3.76[5 \mathrm{H}, \mathrm{s}] ; 4.26[1 \mathrm{H}, \mathrm{s}] 4.44[2 \mathrm{H}, \mathrm{d}, J$ 6]; 6.80-7.00 $[3 \mathrm{H}, \mathrm{m}] ; 7.10-7.30[11 \mathrm{H}, \mathrm{m}] ; 7.44[1 \mathrm{H}$, broad s].

${ }^{13}$ C NMR (75 MHz): $\delta 43.2\left(\mathrm{CH}_{2}\right) ; 52.5\left(\mathrm{CH}_{2}\right) ; 55.2\left(\mathrm{CH}_{3}\right) ; 66.9(\mathrm{CH}) ; 112.8(\mathrm{CH}) ; 113.9(\mathrm{CH})$; $119.6(\mathrm{CH}) ; 127.3(\mathrm{CH}) ; 127.4(\mathrm{CH}) ; 127.6(\mathrm{CH}) ; 128.2(\mathrm{CH}) ; 128.5(\mathrm{CH}) ; 128.6(\mathrm{CH}) ; 129.8$ $(\mathrm{CH}) ; 138.3(\mathrm{C}) ; 139.1(\mathrm{C}) ; 140.7(\mathrm{C}) ; 159.9(\mathrm{C}) ; 171.8(\mathrm{C})$.

GC-MS $13.08 \min (100 \%)$ [226 (80\%), $91(100 \%)]$.

\section{Compound (12d)}

M.W.: 270.33

$[\alpha]_{\mathrm{D}}{ }^{20}:+47.3(c 1.10, \mathrm{MeOH})$

${ }^{1} \mathbf{H}$ NMR $\left(300 \mathrm{MHz}, \mathrm{CDCl}_{3}\right): \delta 1.88[2 \mathrm{H}, \mathrm{s}] ; 3.77[3 \mathrm{H}, \mathrm{s}] ; 4.45[2 \mathrm{H}, \mathrm{ddd}, J 19,15,6] ; 4.55[1 \mathrm{H}, \mathrm{s}]$; 6.80-7.00 [3H, m]; 7.20-7.40 [6H, m]; $7.42[1 \mathrm{H}$, broad s].

${ }^{13}$ C NMR $\left(75 \mathrm{MHz}, \mathrm{CDCl}_{3}\right): \delta 43.3\left(\mathrm{CH}_{2}\right) ; 55.2\left(\mathrm{CH}_{3}\right) ; 59.9(\mathrm{CH}) ; 112.3(\mathrm{CH}) ; 113.7(\mathrm{CH}) ; 119.2$ $(\mathrm{CH}) ; 127.4(\mathrm{CH}) ; 127.7(\mathrm{CH}) ; 128.6(\mathrm{CH}) ; 129.9(\mathrm{CH}) ; 138.3(\mathrm{C}) ; 142.5(\mathrm{C}) ; 159.9(\mathrm{C}) ; 172.7(\mathrm{C})$. GC-MS $9.70 \min (100 \%)$ [270 (<1\%), $136(100 \%), 91(10 \%)]$.

\section{Compound (9e)}

M.W.: 517.02

R.f. $=0.37$ (eluent: $\mathrm{EtOAc} / \mathrm{PE}=3: 7$ )

$[\alpha]_{\mathbf{D}}{ }^{20}:+43.1\left(c 0.93, \mathrm{CHCl}_{3}\right)$

${ }^{1}$ H NMR (300 MHz): $\delta 3.02$ [1H, t, $J$ 4]; $3.43[1 \mathrm{H}, \mathrm{d}, J$ 4]; $3.57[1 \mathrm{H}, \mathrm{d}, J 15] ; 3.61[4 \mathrm{H}, \mathrm{s}] ; 4.24$ [1H, dd, $J 15,4] ; 4.26[1 \mathrm{H}, \mathrm{d}, J 15] ; 4.57[1 \mathrm{H}, \mathrm{s}] ; 4.86[1 \mathrm{H}, \mathrm{dd}, J 15,8] ; 4.97[1 \mathrm{H}, \mathrm{d}, J$ 4]; $6.13[1 \mathrm{H}$, dd, $J 6,2] ; 6.33[1 \mathrm{H}, \mathrm{dd}, J 6,1] ; 7.20-7.40[14 \mathrm{H}, \mathrm{m}] ; 8.18[1 \mathrm{H}, \mathrm{dd}, J 8,4]$.

${ }^{13}$ C NMR $(75 \mathrm{MHz}): \delta 43.1\left(\mathrm{CH}_{2}\right) ; 47.3(\mathrm{CH}) ; 50.4\left(\mathrm{CH}_{2}\right) ; 52.1\left(\mathrm{CH}_{3}\right) ; 64.8(\mathrm{CH}) ; 66.4(\mathrm{CH}) ; 77.8$ $(\mathrm{CH}) ; 80.1(\mathrm{CH}) ; 127.2(\mathrm{CH}) ; 127.4(\mathrm{CH}) ; 127.6(\mathrm{CH}) ; 128.4(\mathrm{CH}) ; 128.5(\mathrm{CH}) ; 128.7(\mathrm{CH}) ; 128.8$ $(\mathrm{CH}) ; 131.5(\mathrm{CH}) ; 134.2(\mathrm{C}) ; 134.5(\mathrm{C}) ; 135.6(\mathrm{CH}) ; 136.4(\mathrm{CH}) ; 138.2(\mathrm{C}) ; 138.6(\mathrm{C}) ; 170.5(\mathrm{C})$; $172.2(\mathrm{C})$.

elem. anal. calc. for $\mathrm{C}_{30} \mathrm{H}_{29} \mathrm{ClN}_{2} \mathrm{O}_{4}$ : C, 69.69, H, 5.65, Cl, 6.86, N, 5.42; found $\mathrm{C}, 69.47, \mathrm{H}, 5.85$, N, 5.68 .

\section{Compound (11e)}

M.W.: 364.87

R.f. $=0.08$ (eluent: $\mathrm{PE} / \mathrm{EtOAc}=6: 4$ )

$[\alpha]_{\mathrm{D}}{ }^{20}:+66.6\left(c 1.10, \mathrm{CHCl}_{3}\right)$

${ }^{1}$ H NMR (300 MHz): $\delta$ 1.60-2.00 [1H, broad s]; $3.76[2 \mathrm{H}, \mathrm{s}] ; 4.28[1 \mathrm{H}, \mathrm{s}] 4.46[2 \mathrm{H}, \mathrm{d}, J$ 6]; 7.10- 
$7.40[14 \mathrm{H}, \mathrm{m}] ; 7.45[1 \mathrm{H}$, broad s].

${ }^{13}$ C NMR (75 MHz): $843.3\left(\mathrm{CH}_{2}\right) ; 52.4\left(\mathrm{CH}_{2}\right) ; 66.2(\mathrm{CH}) ; 127.5(\mathrm{CH}) ; 127.6(\mathrm{CH}) ; 127.7(\mathrm{CH})$; $128.2(\mathrm{CH}) ; 128.6(\mathrm{CH}) ; 128.7(\mathrm{CH}) ; 128.75(\mathrm{CH}) ; 129.0(\mathrm{CH}) ; 134.0(\mathrm{C}) ; 137.6(\mathrm{C}) ; 138.2(\mathrm{C})$; $138.9(\mathrm{C}) ; 171.5(\mathrm{C})$.

GC-MS $13.02 \min (100 \%)$ [230 (60\%), $91(100 \%)]$.

Compound (12e)

M.W.: 274.75

$[\alpha]_{\mathrm{D}}{ }^{20}:+57.4(c 1.65, \mathrm{MeOH})$

${ }^{1} \mathbf{H}$ NMR $\left(300 \mathrm{MHz}, \mathrm{CDCl}_{3}\right): \delta 1.90[2 \mathrm{H}, \mathrm{s}] ; 4.42[2 \mathrm{H}, \mathrm{d}, J$ 6]; 4.50-4.70 [1H, broad s]; 7.20-7.40 $[9 \mathrm{H}, \mathrm{m}] ; 7.48[1 \mathrm{H}, \mathrm{s}]$.

${ }^{13}$ C NMR $\left(75 \mathrm{MHz}, \mathrm{CDCl}_{3}\right): \delta 43.2\left(\mathrm{CH}_{2}\right) ; 59.6(\mathrm{CH}) ; 126.9(\mathrm{CH}) ; 127.4(\mathrm{CH}) ; 127.6(\mathrm{CH}) ; 128.6$ $(\mathrm{CH}) ; 128.8(\mathrm{CH}) ; 128.9(\mathrm{C}) ; 135.5(\mathrm{C}) ; 138.3(\mathrm{C}) ; 172.8(\mathrm{C})$.

GC-MS $9.60 \min (100 \%)$ [274 (1\%), $142(34 \%), 140(100 \%)]$.

\section{Compound (9f)}

M.W.: 496.60

R.f. $=0.35$ (eluent: $\mathrm{EtOAc} / \mathrm{PE}=3: 7$ )

$[\alpha]_{\mathrm{D}}{ }^{20}:+17.5\left(c 1.22, \mathrm{CHCl}_{3}\right)$

${ }^{1}$ H NMR (300 MHz): $\delta 2.90[1 \mathrm{H}, \mathrm{t}, J$ 4]; 2.99 [1H, dd, $J$ 14, 6]; $3.32[1 \mathrm{H}, \mathrm{d}, J$ 4]; 3.38 [1H, dd, $J$ $14,7] ; 3.54[3 \mathrm{H}, \mathrm{s}] ; 3.78[1 \mathrm{H}, \mathrm{dd}, J$ 7, 6]; $4.01[2 \mathrm{H}, \mathrm{dd}, J 27,15] ; 4.30[1 \mathrm{H}, \mathrm{dd}, J 15,5] ; 4.42[1 \mathrm{H}$, $\mathrm{dd}, J 15,6] ; 4.78[1 \mathrm{H}$, broad s]; $4.85[1 \mathrm{H}, \mathrm{d}, J 4] ; 6.30[1 \mathrm{H}, \mathrm{dd}, J 6,1] ; 6.34[1 \mathrm{H}, \mathrm{dd}, J 6,1] ; 6.89$ $[1 \mathrm{H}, \mathrm{t}, J 5] ; 7.10-7.40[15 \mathrm{H}, \mathrm{m}]$.

${ }^{13}$ C NMR (75 MHz): $\delta 35.6\left(\mathrm{CH}_{2}\right) ; 43.2\left(\mathrm{CH}_{2}\right) ; 47.3(\mathrm{CH}) ; 51.4\left(\mathrm{CH}_{2}\right) 51.9\left(\mathrm{CH}_{3}\right) ; 63.6(\mathrm{CH}) ; 64.2$ $(\mathrm{CH}) ; 78.2(\mathrm{CH}) ; 82.7(\mathrm{CH}) ; 126.3(\mathrm{CH}) ; 127.0(\mathrm{CH}) ; 127.3(\mathrm{CH}) ; 127.7(\mathrm{CH}) ; 128.2(\mathrm{CH}) ; 128.3$ $(\mathrm{CH}) ; 128.4(\mathrm{CH}) ; 128.6(\mathrm{CH}) ; 129.3(\mathrm{CH}) ; 135.7(\mathrm{CH}) ; 136.0(\mathrm{CH}) ; 138.2(\mathrm{C}) ; 139.2(\mathrm{C}) ; 139.5$ (C); 172.2 (2C).

elem. anal. calc. for $\mathrm{C}_{31} \mathrm{H}_{32} \mathrm{~N}_{2} \mathrm{O}_{4}$ : C, 74.98, $\mathrm{H}, 6.50, \mathrm{~N}$, 5.64; found $\mathrm{C}, 75.12, \mathrm{H}, 6.36, \mathrm{~N}, 5.37$.

\section{Compound (11f)}

M.W.: 344.45

R.f. $=0.11$ (eluent: $\mathrm{PE} / \mathrm{EtOAc}=6: 4$ )

$[\alpha]_{\mathrm{D}}{ }^{20}:-60.6\left(c 1.46, \mathrm{CHCl}_{3}\right)$

${ }^{1}$ H NMR (300 MHz): $\delta$ 1.60-1.80 [1H, broad s]; 2.77 [1H, dd, $J$ 14, 9]; 3.24 [1H, dd, $J$ 14, 4]; 3.43 $[1 \mathrm{H}, \mathrm{dd}, J 9,4] ; 3.53$ [1H, d, $J 13] ; 3.67[1 \mathrm{H}, \mathrm{d}, J 13] ; 4.42[1 \mathrm{H}, \mathrm{dd}, J 13,4] ; 4.46$ [1H, dd, $J 13,4]$; 6.95-7.00 [2H, m]; 7.10-7.35 [13H, m]; $7.60[1 \mathrm{H}$, broad t, $J$ 5].

${ }^{13}$ C NMR (75 MHz): $839.1\left(\mathrm{CH}_{2}\right) ; 43.0\left(\mathrm{CH}_{2}\right) ; 52.6\left(\mathrm{CH}_{2}\right) ; 63.1(\mathrm{CH}) ; 126.8(\mathrm{CH}) ; 127.1(\mathrm{CH})$; $127.3(\mathrm{CH}) ; 127.6(\mathrm{CH}) ; 127.9(\mathrm{CH}) ; 128.4(\mathrm{CH}) ; 128.6(\mathrm{CH}) ; 128.7(\mathrm{CH}) ; 129.1(\mathrm{CH}) ; 137.2(\mathrm{C})$; 138.3 (C); $139.0(\mathrm{C}) ; 173.3(\mathrm{C})$.

GC-MS $11.97 \min (100 \%)$ [210 (60\%), $91(100 \%)]$.

\section{Compound (12f)}

M.W.: 254.33

$[\alpha]_{\mathbf{D}}{ }^{20}:-58.1\left(c 1.60, \mathrm{CHCl}_{3}\right) ;+16.0(c 1.60, \mathrm{MeOH})$

${ }^{1} \mathbf{H}$ NMR $\left(300 \mathrm{MHz}, \mathrm{CDCl}_{3}\right): \delta 1.40$ [2H, broad s]; 2.75 [1H, dd, $J$ 14, 9]; $3.30[1 \mathrm{H}, \mathrm{dd}, J$ 14, 4]; $3.60-3.70[1 \mathrm{H}, \mathrm{m}] ; 4.44[2 \mathrm{H}, \mathrm{ddd}, J 18,15,6] ; 7.20-7.40[10 \mathrm{H}, \mathrm{m}] ; 7.60[1 \mathrm{H}$, broad s].

${ }^{13}$ C NMR $\left(75 \mathrm{MHz}, \mathrm{CDCl}_{3}\right): \delta 41.0\left(\mathrm{CH}_{2}\right) ; 43.1\left(\mathrm{CH}_{2}\right) ; 56.4(\mathrm{CH}) ; 126.8(\mathrm{CH}) ; 127.4(\mathrm{CH}) ; 127.7$ $(\mathrm{CH}) ; 128.6(\mathrm{CH}) ; 128.7(\mathrm{CH}) ; 129.3(\mathrm{CH}) ; 137.8(\mathrm{C}) ; 138.4(\mathrm{C}) ; 174.0(\mathrm{C})$.

GC-MS $9.17 \min (100 \%)$ [254 (<1\%, M), 120 (100\%), $91(45 \%)]$. 
Compound (12g)

It was obtained analogously to $(\mathbf{1 2 b}) .[\alpha]_{\mathbf{D}}{ }^{20}:-7.0(c 1.40, \mathrm{MeOH})$ 